\title{
Synthesis of chlorinated 3,5-diaryl-2-pyrazolines by the reaction of chlorochalcones with hydrazines
}

\author{
Albert Lévai \\ Department of Organic Chemistry, University of Debrecen, P.O. Box 20, H-4010 Debrecen, \\ Hungary \\ E-mail: alevai@puma.unideb.hu
}

Dedicated to Professor Dr José Elguero on the occasion of his $70^{\text {th }}$ birthday

(received 15 Feb 05; accepted 03 May 05; published on the web 05 May 05)

\begin{abstract}
A series of chlorinated 3,5-diaryl-2-pyrazolines has been synthesized by the reaction of appropriately substituted chlorochalcones and mono-substituted hydrazines in hot acetic acid solution. The structures of all new compounds have been elucidated by microanalysis and ${ }^{1} \mathrm{H}-$ and ${ }^{13} \mathrm{C}$ - NMR spectroscopic measurements.
\end{abstract}

Keywords: Chlorochalcones, hydrazines, 3,5-diaryl-2-pyrazolines

\section{Introduction}

Pyrazolines are well known, and important nitrogen-containing five-membered heterocyclic compounds and various methods have been worked out for their synthesis. ${ }^{1-4}$ Several pyrazoline derivatives have been found to possess considerable biological activities, which stimulated research activity in this field. Their prominent effects are e.g., antimicrobial, ${ }^{5}$ central nervous system, ${ }^{6}$ and immunosuppressive ${ }^{7}$ activities. 2-Pyrazolines seem to be the most frequently studied pyrazoline type compounds. After the pioneering work of Fischer and Knövenagel in the late nineteenth century, ${ }^{8}$ the reaction of $\alpha, \beta$-unsaturated aldehydes and ketones with hydrazines became one of the most popular methods for the preparation of 2-pyrazolines. ${ }^{9-36}$ As a result, numerous substituted 2-pyrazolines have been synthesized, which has made possible structureactivity relationship investigations of these substances. As a continuation of our previous studies in this field, ${ }^{23-25,29,30,34-36}$ we report here the synthesis of new 1-substituted 3,5-diaryl-2pyrazolines which are chlorinated in their aromatic rings. 


\section{Results and Discussion}

Numerous chlorinated organic compounds have various bioactivities which render them valuable active ingredients of medicines or plant protecting agents. As mentioned, pyrazolines possess important pharmacological activities, ${ }^{5-7}$ and therefore they are useful materials in drug research. Taking into consideration the possible beneficial effects of the presence of chlorine atom(s) in an organic compound, it appeared expedient to synthesize a series of systematically chlorinated 2pyrazolines to afford substances for a structure-activity relationship study. Although several chlorinated 2-pyrazolines have already been prepared, ${ }^{15,17,21,27,28,33}$ these compounds were individual members of variously substituted 2-pyrazoline series. For this reason, our aim was to synthesize 1-substituted 3,5-diaryl-2-pyrazolines, chlorinated in their aromatic rings connected to the C-3 and C-5 atoms of the pyrazoline skeleton, by the reaction of chlorochalcones with hydrazines.

The chlorochalcones 1-16 were allowed to react with hydrazine hydrate or phenylhydrazine in hot acetic acid to afford 1-acetyl-3,5-diaryl-2-pyrazolines 17-28 or 3,5-diaryl-1-phenyl-2pyrazolines 29-43 in good yields (65-93\%) (Scheme 1). Utilization of chalcones substituted at the para- position of ring A made possible the synthesis of 3,5-diaryl-2-pyrazolines appropriately substituted at the para-position of their 3-aryl group. The ring B of the starting chalcones 1-16 was mono- or dichlorinated at specific positions. Use of these starting materials made available the preparation of a series of systematically substituted 3,5-diaryl-2-pyrazolines. These new compounds, together with the related known substances, provide useful materials for structureactivity relationship studies.

The structures of all new 2-pyrazolines 17-43 have been elucidated by elemental analyses, ${ }^{1} \mathrm{H}$ - and ${ }^{13} \mathrm{C}-\mathrm{NMR}$ spectroscopic measurements. In the ${ }^{1} \mathrm{H}-\mathrm{NMR}$ spectra of compounds 17-43, the three protons attached to the $\mathrm{C}-4$ and $\mathrm{C}-5$ carbon atoms of the 2-pyrazoline ring gave an ABX spin system. Chemical shifts and the coupling constant values (cf. Experimental Section) unequivocally prove a 2-pyrazoline structure. In the 1-acetyl derivatives 17-28 a singlet signal at about $\delta 2.4$ reveals the presence of the $N$-acetyl group. Singlet signals of the methyl or methoxy substituents have also been assigned in each case. ${ }^{13} \mathrm{C}-\mathrm{NMR}$ chemical shift values of the carbon atoms at 40-45 (C-4), 55-62 (C-5) and about 153 or 146 ppm (C-3) corroborate the 2-pyrazoline character deduced from the ${ }^{1} \mathrm{H}$ - NMR data. The presence of an $\mathrm{N}$-acetyl group is also confirmed by the ${ }^{13} \mathrm{C}$ - chemical shifts of these two carbon atoms detected at about $21\left(\underline{\mathrm{CH}}_{3}\right)$ and $168(\underline{\mathrm{C}}=\mathrm{O})$ ppm.

In conclusion, we have synthesized a systematically substituted series of new, chlorinated 3,5-diaryl-2-pyrazolines for structure-activity relationship studies. The 1-substituted 2pyrazolines are very stable compounds, which renders them beneficial substances for biological or pharmacological trials. 


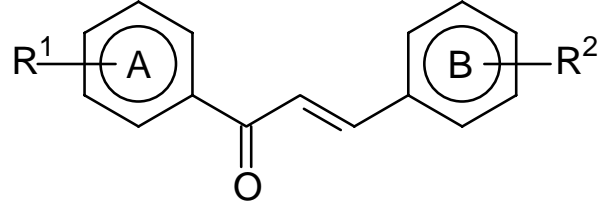

$X=H, P h$

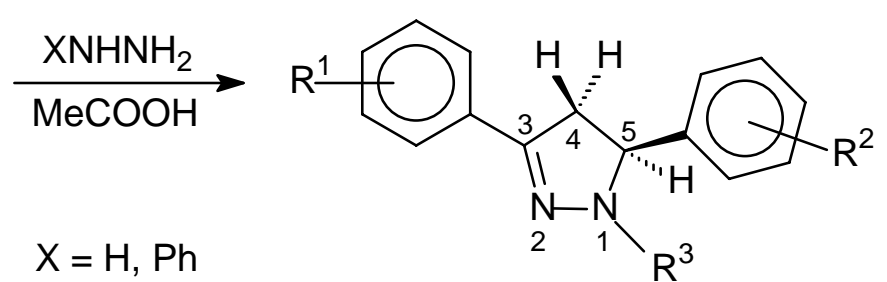

17-43
1, 17: $\mathrm{R}^{1}=\mathrm{H}, \mathrm{R}^{2}=3-\mathrm{Cl}, \mathrm{R}^{3}=\mathrm{AC}$

2, 18: $R^{1}=H, R^{2}=4-C l, R^{3}=A C$

3, 19: $R^{1}=H, R^{2}=2,4-C l_{2}, R^{3}=A c$

4, 20: $R^{1}=H, R^{2}=3,4-C l_{2}, R^{3}=A c$

5, 21: $R^{1}=4-M e, R^{2}=3-C l, R^{3}=A C$

6, 22: $R^{1}=4-M e, R^{2}=4-C l, R^{3}=A C$

7, 23: $R^{1}=4-M e, R^{2}=2,4-C l_{2}, R^{3}=A c$

8, 24: $R^{1}=4-M e, R^{2}=2,6-C l_{2}, R^{3}=A c$

9, 25: $R^{1}=4-\mathrm{MeO}, \mathrm{R}^{2}=2,4-\mathrm{Cl}_{2}, \mathrm{R}^{3}=\mathrm{Ac}$

10, 26: $R^{1}=4-\mathrm{MeO}, \mathrm{R}^{2}=2,6-\mathrm{Cl}_{2}, \mathrm{R}^{3}=\mathrm{Ac}$

11, 27: $R^{1}=4-C l, R^{2}=2,4-C l_{2}, R^{3}=A C$

12, 28: $R^{1}=4-C l, R^{2}=2,6-C l_{2}, R^{3}=A C$

13, 29: $R^{1}=H, R^{2}=2-C l, R^{3}=P h$

2, 30: $R^{1}=H, R^{2}=4-C l, R^{3}=P h$
3, 31: $R^{1}=H, R^{2}=2,4-C l_{2}, R^{3}=P h$

14, 32: $R^{1}=H, R^{2}=2,6-C l_{2}, R^{3}=P h$

4, 33: $R^{1}=H, R^{2}=3,4-C l_{2}, R^{3}=P h$

5, 34: $\mathrm{R}^{1}=4-\mathrm{Me}, \mathrm{R}^{2}=3-\mathrm{Cl}, \mathrm{R}^{3}=\mathrm{Ph}$

6, 35: $\mathrm{R}^{1}=4-\mathrm{Me}, \mathrm{R}^{2}=4-\mathrm{Cl}, \mathrm{R}^{3}=\mathrm{Ph}$

7, 36: $R^{1}=4-\mathrm{Me}, \mathrm{R}^{2}=2,4-\mathrm{Cl}_{2}, \mathrm{R}^{3}=\mathrm{Ph}$

8, 37: $R^{1}=4-\mathrm{Me}, \mathrm{R}^{2}=2,6-\mathrm{Cl}_{2}, \mathrm{R}^{3}=\mathrm{Ph}$

9, 38: $\mathrm{R}^{1}=4-\mathrm{MeO}, \mathrm{R}^{2}=2,4-\mathrm{Cl}_{2}, \mathrm{R}^{3}=\mathrm{Ph}$

10, 39: $\mathrm{R}^{1}=4-\mathrm{MeO}, \mathrm{R}^{2}=2,6-\mathrm{Cl}_{2}, \mathrm{R}^{3}=\mathrm{Ph}$

15, 40: $R^{1}=4-F, R^{2}=3,4-C l_{2}, R^{3}=P h$

11, 41: $\mathrm{R}^{1}=4-\mathrm{Cl}, \mathrm{R}^{2}=2,4-\mathrm{Cl}_{2}, \mathrm{R}^{3}=\mathrm{Ph}$

12, 42: $\mathrm{R}^{1}=4-\mathrm{Cl}, \mathrm{R}^{2}=2,6-\mathrm{Cl}_{2}, \mathrm{R}^{3}=\mathrm{Ph}$

16, 43: $\mathrm{R}^{1}=4-\mathrm{Cl}, \mathrm{R}^{2}=3,4-\mathrm{Cl}_{2}, \mathrm{R}^{3}=\mathrm{Ph}$

\section{Scheme 1}

\section{Experimental Section}

General Procedures. Melting points were determined with a Kofler hot-stage apparatus and are uncorrected. ${ }^{1} \mathrm{H}$ - and ${ }^{13} \mathrm{C}$ - NMR spectra were recorded on a Varian Gemini 200 spectrometer at 200/50 $\mathrm{MHz}$ in $\mathrm{CDCl}_{3}$ (internal standard TMS, $\delta=0.0 \mathrm{ppm}$ ) at ambient temperature $\left(\mathrm{ca} 20{ }^{\circ} \mathrm{C}\right.$ ). Elemental analyses were measured in-house with a Carlo Erba 1106 EA instrument. TLC was performed on Kieselgel $60 \mathrm{~F}_{254}$ (Merck) layer using toluene:ethyl acetate $(4: 1 \mathrm{v} / \mathrm{v})$ or hexane:acetone $(7: 3 \mathrm{v} / \mathrm{v})$ as eluents. Starting materials 1-16 were synthesized according to known procedures. ${ }^{37-40}$ 


\section{General procedure for the preparation of compounds 17-43}

A mixture of chlorochalcone (1-16, 10.0 mmoles), hydrazine hydrate $(50.0 \mathrm{mmoles})$ or phenyl hydrazine $(50.0$ mmoles $)$ and acetic acid $(60 \mathrm{~mL})$ was heated at reflux for $3 \mathrm{~h}$, then poured onto crushed ice. The precipitate was separated by filtration, washed with water, and crystallized from methanol to obtain the 2-pyrazolines 17-43 (Scheme 1).

1-Acetyl-5-(3-chlorophenyl)-3-phenyl-2-pyrazoline (17). Obtained as white needles in 90\% yield, mp 120-121 ${ }^{\circ} \mathrm{C} ;{ }^{1} \mathrm{H}$ NMR $(\delta): 2.45$ (3H, s, Me), $3.10\left(1 \mathrm{H}, \mathrm{dd}, \mathrm{J}=5.2,17.8 \mathrm{~Hz}, 4-\mathrm{H}_{\text {trans }}\right.$ ), $3.77\left(1 \mathrm{H}, \mathrm{dd}, \mathrm{J}=11.4,17.8 \mathrm{~Hz}, 4-\mathrm{H}_{\mathrm{cis}}\right), 5.53(1 \mathrm{H}, \mathrm{dd}, \mathrm{J}=5.2,11.4 \mathrm{~Hz}, 5-\mathrm{H}), 7.10-7.76(\mathrm{~m}, 9 \mathrm{x}$ arom. H); ${ }^{13} \mathrm{C}-\mathrm{NMR}(\delta): 21.9,42.2,59.4,123.8,125.7,126.6,127.8,128.7,130.2,130.4,131.1$, 134.7, 143.8, 153.6, 168.8; Anal. Calcd. for $\mathrm{C}_{17} \mathrm{H}_{15} \mathrm{ClN}_{2} \mathrm{O}: \mathrm{C}, 68.34 ; \mathrm{H}, 5.06$; N, 9.37. Found: $\mathrm{C}$, $68.46 ; \mathrm{H}, 5.11 ; \mathrm{N}, 9.28 \%$.

1-Acetyl-5-(4-chlorophenyl)-3-phenyl-2-pyrazoline (18). Isolated as white needles in 67\% yield, mp 108-109 ${ }^{\circ} \mathrm{C} ;{ }^{1} \mathrm{H}$ NMR $(\delta): 2.41(3 \mathrm{H}, \mathrm{s}, \mathrm{Me}), 3.13\left(1 \mathrm{H}, \mathrm{dd}, \mathrm{J}=5.0,18.1 \mathrm{~Hz}, 4-\mathrm{H}_{\text {trans }}\right)$, $3.76\left(1 \mathrm{H}, \mathrm{dd}, \mathrm{J}=10.8,18.1 \mathrm{~Hz}, 4-\mathrm{H}_{\text {cis }}\right), 5.66(1 \mathrm{H}, \mathrm{dd}, \mathrm{J}=5.0,10.8 \mathrm{~Hz}, 5-\mathrm{H}), 7.14-7.75(\mathrm{~m}, 9 \mathrm{x}$

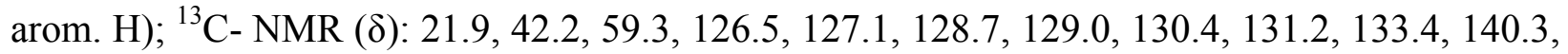
153.6, 168.8; Anal. Calcd. for $\mathrm{C}_{17} \mathrm{H}_{15} \mathrm{ClN}_{2} \mathrm{O}$ : C, 68.34; H, 5.06; N, 9.37. Found: C, 68.24; H, $5.02 ; \mathrm{N}, 9.48 \%$.

1-Acetyl-5-(2,4-dichlorophenyl)-3-phenyl-2-pyrazoline (19). Obtained as white plates, in 74\% yield, mp 137-138 ${ }^{\circ} \mathrm{C}$; ${ }^{1} \mathrm{H}-\mathrm{NMR}(\delta): 2.47(3 \mathrm{H}, \mathrm{s}, \mathrm{Me}), 3.04\left(1 \mathrm{H}, \mathrm{dd}, \mathrm{J}=4.9,17.6 \mathrm{~Hz}, 4-\mathrm{H}_{\text {trans }}\right)$, $3.83\left(1 \mathrm{H}, \mathrm{dd}, \mathrm{J}=10.8,17.6 \mathrm{~Hz}, 4-\mathrm{H}_{\text {cis }}\right), 5.84(1 \mathrm{H}, \mathrm{dd}, \mathrm{J}=4.9,10.8 \mathrm{~Hz}, 5-\mathrm{H}), 6.98-7.75$ (m, 8x

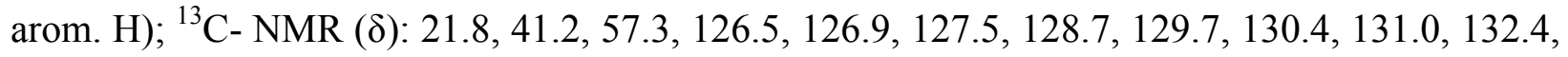
133.8, 137.2, 154.0, 168.8; Anal. Calcd. for $\mathrm{C}_{17} \mathrm{H}_{14} \mathrm{Cl}_{2} \mathrm{~N}_{2} \mathrm{O}$ : C, 61.27; H, 4.23; N, 8.40. Found: C, $61.40 ; \mathrm{H}, 4.28 ; \mathrm{N}, 8.49 \%$.

1-Acetyl-5-(3,4-dichlorophenyl)-3-phenyl-2-pyrazoline (20). Prepared as white plates in 70\% yield, mp $134-135{ }^{\circ} \mathrm{C} ;{ }^{1} \mathrm{H}-\mathrm{NMR}(\delta): 2.42(3 \mathrm{H}, \mathrm{s}, \mathrm{Me}), 3.12\left(1 \mathrm{H}, \mathrm{dd}, \mathrm{J}=4.9,17.8 \mathrm{~Hz}, 4-\mathrm{H}_{\text {trans }}\right)$, $3.76\left(1 \mathrm{H}, \mathrm{dd}, \mathrm{J}=11.8,17.8 \mathrm{~Hz}, 4-\mathrm{H}_{\mathrm{cis}}\right), 5.52(1 \mathrm{H}, \mathrm{dd}, \mathrm{J}=4.9,11.8 \mathrm{~Hz}, 5-\mathrm{H}), 7.06-7.75(\mathrm{~m}, 8 \mathrm{x}$ arom. H); ${ }^{13} \mathrm{C}-\mathrm{NMR}(\delta): 21.8,42.1,59.0,125.2,126.6,127.7,128.8,130.5,130.9,131.7,133.0$, 142.0, 153.6, 168.9; Anal. Calcd. for $\mathrm{C}_{17} \mathrm{H}_{14} \mathrm{Cl}_{2} \mathrm{~N}_{2} \mathrm{O}$ : C, 61.27; H, 4.23; N, 8.40. Found: C, $61.16 ; \mathrm{H}, 4.18 ; \mathrm{N}, 8.46 \%$.

1-Acetyl-5-(3-chlorophenyl)-3-(4-methylphenyl)-2-pyrazoline (21). Obtained as white needles in 78\% yield, mp $152-153{ }^{\circ} \mathrm{C}$; ${ }^{1} \mathrm{H}-\mathrm{NMR}(\delta): 2.39(3 \mathrm{H}, \mathrm{s}, \mathrm{Me}), 2.43(3 \mathrm{H}, \mathrm{s}, \mathrm{Me}), 3.12(1 \mathrm{H}, \mathrm{dd}, \mathrm{J}$ $\left.=4.8,17.7 \mathrm{~Hz}, 4-\mathrm{H}_{\text {trans }}\right), 3.73\left(1 \mathrm{H}, \mathrm{dd}, \mathrm{J}=11.8,17.7 \mathrm{~Hz}, 4-\mathrm{H}_{\mathrm{cis}}\right), 5.53(1 \mathrm{H}, \mathrm{dd}, \mathrm{J}=4.8,11.8 \mathrm{~Hz}$, 5-H), 7.09-7.63 (m, 8x arom. H); ${ }^{13} \mathrm{C}-\mathrm{NMR}(\delta): 21.4,21.8,42.2,59.3,123.8,125.7,126.5$, 127.7, 128.4, 129.4, 130.1, 134.7, 140.7, 143.9, 153.7, 168.7; Anal. Calcd. for $\mathrm{C}_{18} \mathrm{H}_{17} \mathrm{ClN}_{2} \mathrm{O}$ : C, 69.12; H, 5.48; N, 8.95. Found: C, 69.04; H, 5.53; N, 8.89\%.

1-Acetyl-5-(4-chlorophenyl)-3-(4-methylphenyl)-2-pyrazoline (22). Isolated as white needles in $68 \%$ yield, mp $127-128{ }^{\circ} \mathrm{C} ;{ }^{1} \mathrm{H}-\mathrm{NMR}(\delta): 2.38(3 \mathrm{H}, \mathrm{s}, \mathrm{Me}), 2.42(3 \mathrm{H}, \mathrm{s}, \mathrm{Me}), 3.10(1 \mathrm{H}, \mathrm{dd}, \mathrm{J}$ $\left.=4.8,17.8 \mathrm{~Hz}, 4-\mathrm{H}_{\text {trans }}\right), 3.74\left(1 \mathrm{H}, \mathrm{dd}, \mathrm{J}=11.8,17.8 \mathrm{~Hz}, 4-\mathrm{H}_{\text {cis }}\right), 5.54(1 \mathrm{H}, \mathrm{dd}, \mathrm{J}=4.8,11.8 \mathrm{~Hz}$, 5-H), 7.13-7.62 (m, 8x arom. H); ${ }^{13} \mathrm{C}-\mathrm{NMR}(\delta): 21.4,21.8,42.2,59.2,126.5,127.0,128.4$, 
128.9, 129.4, 133.3, 140.4, 140.7, 153.7, 168.7; Anal. Calcd. for $\mathrm{C}_{18} \mathrm{H}_{17} \mathrm{ClN}_{2} \mathrm{O}: \mathrm{C}, 69.12 ; \mathrm{H}$, 5.48; N, 8.95. Found: $69.26 ; \mathrm{H}, 5.42 ; \mathrm{N}, 8.86 \%$.

1-Acetyl-5-(2,4-dichlorophenyl)-3-(4-methylphenyl)-2-pyrazoline (23). Prepared as white plates in 69\% yield, mp $187-188{ }^{\circ} \mathrm{C} ;{ }^{1} \mathrm{H}-\mathrm{NMR}(\delta): 2.38(3 \mathrm{H}, \mathrm{s}, \mathrm{Me}), 2.46(3 \mathrm{H}, \mathrm{s}, \mathrm{Me}), 2.98(1 \mathrm{H}$, $\left.\mathrm{dd}, \mathrm{J}=5.0,17.8 \mathrm{~Hz}, 4-\mathrm{H}_{\text {trans }}\right), 3.80\left(1 \mathrm{H}, \mathrm{dd}, \mathrm{J}=11.9,17.8 \mathrm{~Hz}, 4-\mathrm{H}_{\text {cis }}\right), 5.82(1 \mathrm{H}, \mathrm{dd}, \mathrm{J}=5.0,11.9$ $\mathrm{Hz}, 5-\mathrm{H}), 6.98-7.62$ (m, 7x arom. H); ${ }^{13} \mathrm{C}-\mathrm{NMR}(\delta): 21.4,21.8,41.3,57.2,126.5,126.9,127.5$, 128.3, 129.4, 129.7, 132.4, 133.8, 137.3, 140.9, 154.2, 168.8; Anal. Calcd. for $\mathrm{C}_{18} \mathrm{H}_{16} \mathrm{Cl}_{2} \mathrm{~N}_{2} \mathrm{O}$ : C, 62.26; H, 4.64; N, 8.06. Found: C, 62.39; H, 4.69; N, 8.13\%.

1-Acetyl-5-(2,6-dichlorophenyl)-3-(4-methylphenyl)-2-pyrazoline (24). Prepared as pale yellow needles in $68 \%$ yield, mp $198-199{ }^{\circ} \mathrm{C} ;{ }^{1} \mathrm{H}-\mathrm{NMR}(\delta): 2.37(3 \mathrm{H}, \mathrm{s}, \mathrm{Me}), 2.41$ (3H, s, Me), $3.28\left(1 \mathrm{H}, \mathrm{dd} \mathrm{J}=7.6,17.8 \mathrm{~Hz}, 4-\mathrm{H}_{\text {trans }}\right), 3.67\left(1 \mathrm{H}, \mathrm{dd}, \mathrm{J}=13.0,17.8 \mathrm{~Hz}, 4-\mathrm{H}_{\text {cis }}\right), 6.21(1 \mathrm{H}, \mathrm{dd}, \mathrm{J}=$ 7.6, $13.0 \mathrm{~Hz}, 5-\mathrm{H}), 7.08-7.66\left(\mathrm{~m}, 7 \mathrm{x}\right.$ arom. H); ${ }^{13} \mathrm{C}-\mathrm{NMR}(\delta): 21.4,21.6,39.1,56.6,126.4$, 128.4, 128.6, 128.8, 129.4, 129.8, 133.6, 135.1, 135.8, 140.5, 153.5, 168.8; Anal. Calcd. for $\mathrm{C}_{18} \mathrm{H}_{16} \mathrm{Cl}_{2} \mathrm{~N}_{2} \mathrm{O}$ : C, 62.26; H, 4.64; N, 8.06. Found: C, 62.17; H, 4.59; N, 7.97\%.

1-Acetyl-5-(2,4-dichlorophenyl)-3-(4-methoxyphenyl)-2-pyrazoline (25). Obtained as white plates in 75\% yield, mp 170-171 ${ }^{\circ} \mathrm{C} ;{ }^{1} \mathrm{H}-\mathrm{NMR}(\delta): 2.47(3 \mathrm{H}, \mathrm{s}, \mathrm{Me}), 2.99(1 \mathrm{H}, \mathrm{dd}, \mathrm{J}=4.9,17.7$ $\left.\mathrm{Hz}, 4-\mathrm{H}_{\text {trans }}\right), 3.81\left(1 \mathrm{H}, \mathrm{dd}, \mathrm{J}=11.8,17.7 \mathrm{~Hz}, 4-\mathrm{H}_{\mathrm{cis}}\right), 3.86(3 \mathrm{H}, \mathrm{s}, \mathrm{MeO}), 5.84(1 \mathrm{H}, \mathrm{dd}, \mathrm{J}=4.9$, $11.8 \mathrm{~Hz}, 5-\mathrm{H}), 6.90-7.70$ (m, 7x arom. H); ${ }^{13} \mathrm{C}-\mathrm{NMR}(\delta): 21.8,41.3,55.3,57.1,123.6,126.9$, $127.5,128.1,129.7,132.4,133.7,137.3,153.8,161.4,168.6$; Anal. Calcd. for $\mathrm{C}_{18} \mathrm{H}_{16} \mathrm{Cl}_{2} \mathrm{~N}_{2} \mathrm{O}_{2}$ : C, 59.52; H, 4.44; N, 7.71. Found: C, 59.64; H, 4.39; N, 7.78\%.

1-Acetyl-5-(2,6-dichlorophenyl)-3-(4-methoxyphenyl)-2-pyrazoline (26). Prepared as pale yellow plates in $69 \%$ yield, mp $176-177{ }^{\circ} \mathrm{C} ;{ }^{1} \mathrm{H}-\mathrm{NMR}(\delta): 2.35(3 \mathrm{H}, \mathrm{s}, \mathrm{Me}), 3.25(1 \mathrm{H}, \mathrm{dd}, \mathrm{J}=$ 8.4, $\left.17.7 \mathrm{~Hz}, 4-\mathrm{H}_{\text {trans }}\right), 3.64\left(1 \mathrm{H}, \mathrm{dd}, \mathrm{J}=12.8,17.7 \mathrm{~Hz}, 4-\mathrm{H}_{\text {cis }}\right), 3.83(3 \mathrm{H}, \mathrm{s}, \mathrm{MeO}), 6.19$ (1H, dd, J $=8.4,12.8 \mathrm{~Hz}, 5-\mathrm{H}), 6.95-7.70\left(\mathrm{~m}, 7 \mathrm{x}\right.$ arom. H); ${ }^{13} \mathrm{C}-\mathrm{NMR}(\delta): 21.5,39.1,55.3,56.5,123.9$, $128.0,128.4,128.8,129.8,133.6,135.1,135.8,153.2,161.2,168.7$; Anal. Calcd. for $\mathrm{C}_{18} \mathrm{H}_{16} \mathrm{Cl}_{2} \mathrm{~N}_{2} \mathrm{O}_{2}$ : C, 59.52; H, 4.44; N, 7.71. Found: C, 59.41; H, 4.49; N, 7.80\%.

1-Acetyl-3-(4-chlorophenyl)-5-(2,4-dichlorophenyl)-2-pyrazoline (27). Isolated as white plates in 76\% yield, mp $131-132{ }^{\circ} \mathrm{C} ;{ }^{1} \mathrm{H}-\mathrm{NMR}(\delta): 2.41(3 \mathrm{H}, \mathrm{s}, \mathrm{Me}), 3.10(1 \mathrm{H}, \mathrm{dd}, \mathrm{J}=5.1,17.9$ $\left.\mathrm{Hz}, 4-\mathrm{H}_{\text {trans }}\right), 4.74\left(1 \mathrm{H}, \mathrm{dd}, \mathrm{J}=12.0,17.9 \mathrm{~Hz}, 4-\mathrm{H}_{\mathrm{cis}}\right), 5.52(1 \mathrm{H}, \mathrm{dd}, \mathrm{J}=5.1,12.0 \mathrm{~Hz}, 5-\mathrm{H}), 7.05-$ 7.67 (m, 7x arom. H); ${ }^{13} \mathrm{C}$ - NMR $(\delta): 21.9,42.1,59.3,125.2,127.8,127.9,129.2,129.6,131.0$, 131.9, 133.1, 136.7, 152.6, 169.0; Anal. Calcd. for $\mathrm{C}_{17} \mathrm{H}_{13} \mathrm{Cl}_{3} \mathrm{~N}_{2} \mathrm{O}$ : C, 55.53; H, 3.56; N, 7.62. Found: C, 55.62; H, 3.52; N, 7.72\%.

1-Acetyl-3-(4-chlorophenyl)-5-(2,6-dichlorophenyl)-2-pyrazoline (28). Obtained as pale yellow plates in $80 \%$ yield, mp $188-189{ }^{\circ} \mathrm{C} ;{ }^{1} \mathrm{H}-\mathrm{NMR}(\delta): 2.36(3 \mathrm{H}, \mathrm{s}, \mathrm{Me}), 3.26(1 \mathrm{H}, \mathrm{dd}, \mathrm{J}=$ 8.4, $\left.17.7 \mathrm{~Hz}, 4-\mathrm{H}_{\text {trans }}\right), 3.67\left(1 \mathrm{H}, \mathrm{dd}, \mathrm{J}=13.0,17.7 \mathrm{~Hz}, 4-\mathrm{H}_{\mathrm{cis}}\right), 6.23(1 \mathrm{H}$, dd, J = 8.4, $13.0 \mathrm{~Hz}, 5-$ H), 7.10-7.70 (m, 7x arom. H); ${ }^{13} \mathrm{C}-\mathrm{NMR}(\delta): 21.5,38.9,56.7,127.7,128.4,128.9,129.8,133.5$, 134.8, 135.8, 136.1, 152.3, 168.8; Anal. Calcd. for $\mathrm{C}_{17} \mathrm{H}_{13} \mathrm{Cl}_{3} \mathrm{~N}_{2} \mathrm{O}$ : C, 55.53; H, 3.56; N, 7.62. Found: C, 55.46; H, 3.61; N, 7.71\%.

5-(2-Chlorophenyl)-1,3-diphenyl-2-pyrazoline (29). Isolated as pale yellow needles in 90\% yield, mp 141-142 ${ }^{\circ} \mathrm{C} ;{ }^{1} \mathrm{H}-\mathrm{NMR}(\delta): 3.06\left(1 \mathrm{H}, \mathrm{dd}, \mathrm{J}=4.9,17.6 \mathrm{~Hz}, 4-\mathrm{H}_{\text {trans }}\right), 3.96(1 \mathrm{H}, \mathrm{dd}, \mathrm{J}=$ 
$\left.11.4,17.6 \mathrm{~Hz}, 4-\mathrm{H}_{\mathrm{cis}}\right), 5.64(1 \mathrm{H}, \mathrm{dd}, \mathrm{J}=4.9,11.4 \mathrm{~Hz}, 5-\mathrm{H}), 6.76-7.74\left(\mathrm{~m}, 14 \mathrm{x}\right.$ arom. H); ${ }^{13} \mathrm{C}-$ NMR $(\delta): 41.9,61.2,113.1,119.2,125.7,127.3,127.6,128.5,128.6,128.7,129.0 .129 .8,131.7$, 132.6, 139.2, 144.4, 147.0; Anal. Calcd. for $\mathrm{C}_{21} \mathrm{H}_{17} \mathrm{ClN}_{2}$ : C, 75.78; H, 5.15; N, 8.41. Found: C, 75.91; H, 5.19; N, 8.34\%.

5-(4-Chlorophenyl)-1,3-diphenyl-2-pyrazoline (30). Prepared as pale yellow needles in 93\% yield, mp 133-134 ${ }^{\circ} \mathrm{C} ;{ }^{1} \mathrm{H}-\mathrm{NMR}(\delta): 3.04\left(1 \mathrm{H}, \mathrm{dd}, \mathrm{J}=7.4,17.6 \mathrm{~Hz}, 4-\mathrm{H}_{\text {trans }}\right), 3.77(1 \mathrm{H}, \mathrm{dd}, \mathrm{J}=$ 11.6, 17.6 Hz, 4- $\left.\mathrm{H}_{\text {cis }}\right), 5.67(1 \mathrm{H}, \mathrm{dd}, \mathrm{J}=7.4,11.6 \mathrm{~Hz}, 5-\mathrm{H}), 6.71-7.64(\mathrm{~m}, 14 \mathrm{x}$ arom. $\mathrm{H}) ;{ }^{13} \mathrm{C}-$ NMR $(\delta): 43.4,63.8,113.4,119.4,125.7,127.3,128.5,128.7,128.9,129.3,129.9,132.5,133.3$, 141.1, 144.6, 146.7; Anal. Calcd. for $\mathrm{C}_{21} \mathrm{H}_{17} \mathrm{ClN}_{2}$ : C, 75.78; H, 5.15; N, 8.41. Found: C, 75.69; H; 5.10; N, 8.49\%.

5-(2,4-Dichlorophenyl)-1,3-diphenyl-2-pyrazoline (31). Obtained as yellow plates in 76\% yield, mp 119-120 ${ }^{\circ} \mathrm{C} ;{ }^{1} \mathrm{H}-\mathrm{NMR}(\delta): 3.02\left(1 \mathrm{H}, \mathrm{dd}, \mathrm{J}=6.7,17.3 \mathrm{~Hz}, 4-\mathrm{H}_{\text {trans }}\right), 3.97(1 \mathrm{H}, \mathrm{dd}, \mathrm{J}=$ $\left.12.3,17.3 \mathrm{~Hz}, 4-\mathrm{H}_{\mathrm{cis}}\right), 5.59(1 \mathrm{H}, \mathrm{dd}, \mathrm{J}=6.7,12.3 \mathrm{~Hz}, 5-\mathrm{H}), 6.69-7.71(\mathrm{~m}, 13 \mathrm{x}$ arom. $\mathrm{H}) ;{ }^{13} \mathrm{C}-$

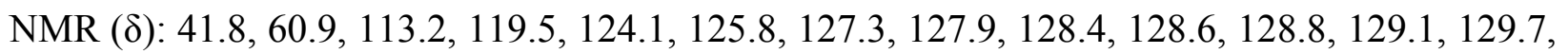
132.4, 133.9, 137.9, 144.2, 147.1; Anal. Calcd. for $\mathrm{C}_{21} \mathrm{H}_{16} \mathrm{Cl}_{2} \mathrm{~N}_{2}$ : C, 68.67; H, 4.39; N, 7.62. Found: C, 68.78; H, 4.34; N, 7.68\%.

5-(2,6-Dichlorophenyl)-1,3-diphenyl-2-pyrazoline (32). Prepared as yellow needles in 65\% yield, mp 191-192 ${ }^{\circ} \mathrm{C} ;{ }^{1} \mathrm{H}-\mathrm{NMR}(\delta): 3.30\left(1 \mathrm{H}, \mathrm{dd}, \mathrm{J}=9.6,17.3 \mathrm{~Hz}, 4-\mathrm{H}_{\text {trans }}\right), 3.76(1 \mathrm{H}, \mathrm{dd}, \mathrm{J}=$ 13.6, $\left.17.3 \mathrm{~Hz}, 4-\mathrm{H}_{\text {cis }}\right), 6.03(1 \mathrm{H}, \mathrm{dd}, \mathrm{J}=9.6,13.6 \mathrm{~Hz}, 5-\mathrm{H}), 6.73-7.76$ (m, 13x arom. H); ${ }^{13} \mathrm{C}-$ NMR $(\delta): 39.9,60.0,113.1,119.2,125.7,128.5,128.9,129.2,130.8,132.6,135.0,135.2,135.8$, 144.6, 146.4; Anal. Calcd. for $\mathrm{C}_{21} \mathrm{H}_{16} \mathrm{Cl}_{2} \mathrm{~N}_{2}$ : C, 68.67; $\mathrm{H}, 4.39 ; \mathrm{N}, 7.62$. Found: C, 68.58; $\mathrm{H}$, $4.45 ; \mathrm{N}, 7.71 \%$.

5-(3,4-Dichlorophenyl)-1,3-diphenyl-2-pyrazoline (33). Obtained as yellow needles in 79\% yield, mp 109-110 ${ }^{\circ} \mathrm{C},{ }^{1} \mathrm{H}-\mathrm{NMR}(\delta): 3.08\left(1 \mathrm{~h}, \mathrm{dd}, \mathrm{J}=7.2,17.2 \mathrm{~Hz}, 4-\mathrm{H}_{\text {trans }}\right), 3.80(1 \mathrm{H}, \mathrm{dd}, \mathrm{J}=$ $\left.12.4,17.2 \mathrm{~Hz}, 4-\mathrm{H}_{\mathrm{cis}}\right), 5.19(1 \mathrm{H}, \mathrm{dd}, \mathrm{J}=7.2,12.4 \mathrm{~Hz}, 5-\mathrm{H}), 6.79-7.72$ (m, 13x arom. H); ${ }^{13} \mathrm{C}-$ NMR $(\delta): 43.4,63.5,105.5,119.6,125.3,125.4,125.8,127.9,128.3,128.6,128.9,129.0,129.2$, 130.3, 131.2, 132.3, 133.2, 142.9, 144.5, 146.8; Anal. Calcd. for $\mathrm{C}_{21} \mathrm{H}_{16} \mathrm{Cl}_{2} \mathrm{~N}_{2}$ : C, 68.67; H, 4.39; N, 7.62. Found: C, 68.61; H, 4.43; N, 7.54\%.

5-(3-Chlorophenyl)-3-(4-methylphenyl)-1-phenyl-2-pyrazoline (34). Isolated as pale yellow plates in $72 \%$ yield, mp $127-128{ }^{\circ} \mathrm{C} ;{ }^{1} \mathrm{H}-\mathrm{NMR}(\delta): 2.42(3 \mathrm{H}, \mathrm{s}, \mathrm{Me}), 3.04(1 \mathrm{H}, \mathrm{dd}, \mathrm{J}=5.1,17.7$ $\left.\mathrm{Hz}, 4-\mathrm{H}_{\text {trans }}\right), 3.87\left(1 \mathrm{H}, \mathrm{dd}, \mathrm{J}=11.2,17.7 \mathrm{~Hz}, 4-\mathrm{H}_{\mathrm{cis}}\right), 5.23(1 \mathrm{H}, \mathrm{dd}, \mathrm{J}=5.1,11.2 \mathrm{~Hz}, 5-\mathrm{H}), 6.84$ 7.67 (m, 13x arom. H); ${ }^{13} \mathrm{C}-\mathrm{NMR}(\delta): 21.3,43.6,63.9,113.3,119.2,124.0,125.4,125.7,126.1$, $127.8,128.9,129.2,129.7,130.4,134.9,138.8,144.9$, 146.9; Anal. Calcd. for $\mathrm{C}_{22} \mathrm{H}_{19} \mathrm{ClN}_{2}$ : C, 76.18; H, 5.52; N, 8.07. Found: C, 76.29; H, 5.47; N, 8.16\%.

5-(4-Chlorophenyl)-3-(4-methylphenyl)-1-phenyl-2-pyrazoline (35). Prepared as pale yellow plates in 75\% yield, mp $155-156{ }^{\circ} \mathrm{C} ;{ }^{1} \mathrm{H}-\mathrm{NMR} \delta 2.42(3 \mathrm{H}, \mathrm{s}, \mathrm{Me}), 3.13(1 \mathrm{H}, \mathrm{dd}, \mathrm{J}=5.3,17.8$ $\left.\mathrm{Hz}, 4-\mathrm{H}_{\text {trans }}\right), 3.87\left(1 \mathrm{H}, \mathrm{dd}, \mathrm{J}=11.5,17.8 \mathrm{~Hz}, 4-\mathrm{H}_{\mathrm{cis}}\right), 5.28(1 \mathrm{H}, \mathrm{dd}, \mathrm{J}=5.3,11.5 \mathrm{~Hz}, 5-\mathrm{H}), 6.80$ 7.67 (m, 13x arom. H); ${ }^{13} \mathrm{C}-\mathrm{NMR}(\delta): 21.3,43.6,63.8,105.1,113.4,119.2,124.8,125.5,125.7$, $125.9,127.3,128.8,128.9,129.4,130.5,133.3,134.5,138.8,144.8,146.9$; Anal. Calcd. for $\mathrm{C}_{22} \mathrm{H}_{19} \mathrm{ClN}_{2}$ : C, 76.18; H, 5.52; N, 8.07. Found: 76.08; H, 5.59; N, 7.93\%. 
5-(2,4-Dichlorophenyl)-3-(4-methylphenyl)-1-phenyl-2-pyrazoline (36). Obtained as yellow needles in 76\% yield, mp $130-131{ }^{\circ} \mathrm{C} ;{ }^{1} \mathrm{H}-\mathrm{NMR}(\delta): 2.39(3 \mathrm{H}, \mathrm{s}, \mathrm{Me}), 3.03(1 \mathrm{H}, \mathrm{dd}, \mathrm{J}=4.9,17.8$ $\left.\mathrm{Hz}, 4-\mathrm{H}_{\text {trans }}\right), 3.98\left(1 \mathrm{H}, \mathrm{dd}, \mathrm{J}=9.8,17.8 \mathrm{~Hz}, 4-\mathrm{H}_{\mathrm{cis}}\right), 5.60(1 \mathrm{H}, \mathrm{dd}, \mathrm{J}=4.9,9.8 \mathrm{~Hz}, 5-\mathrm{H}), 6.70-$ 7.67 (m, 12x arom. H); ${ }^{13} \mathrm{C}-\mathrm{NMR}(\delta): 21.4,41.9,60.8,113.1,119.3,125.8,127.9,128.5,129.1$, 129.3, 129.7, 132.4, 133.8, 138.0, 139.0, 144.4, 147.3; Anal. Calcd. for $\mathrm{C}_{22} \mathrm{H}_{18} \mathrm{Cl}_{2} \mathrm{~N}_{2}$ : C, 69.30; H, 4.76; N, 7.34. Found: C, 69.38; H, 4.81; N, 7.25\%.

5-(2,6-Dichlorophenyl)-3-(4-methylphenyl)-1-phenyl-2-pyrazoline (37). Isolated as pale yellow needles in $76 \%$ yield, mp $185-186{ }^{\circ} \mathrm{C}$; ${ }^{1} \mathrm{H}-\mathrm{NMR}(\delta): 2.44(3 \mathrm{H}, \mathrm{s}, \mathrm{Me}), 3.38(1 \mathrm{H}, \mathrm{dd}, \mathrm{J}=$ 9.4, $\left.17.5 \mathrm{~Hz}, 4-\mathrm{H}_{\text {trans }}\right), 3.82\left(1 \mathrm{H}, \mathrm{dd}, \mathrm{J}=13.4,17.5 \mathrm{~Hz}, 4-\mathrm{H}_{\mathrm{cis}}\right), 6.09$ (1H, dd, J = 9.4. 13.4 Hz, 5H), 6.80-7.69 (m, 12x arom. H); ${ }^{13} \mathrm{C}-\mathrm{NMR}(\delta): 21.4,40.0,59.9,113.0,119.0,125.7,128.4$, $128.9,129.2,129.8,130.8,135.0,135.2,135.9,138.6,144.8,146.5$; Anal. Calcd. for $\mathrm{C}_{22} \mathrm{H}_{18} \mathrm{Cl}_{2} \mathrm{~N}_{2}$ : C, 69.30; H, 4.76; N, 7.34. Found: C, 69.21; H, 4.71; N, 3.44\%.

5-(2,4-Dichlorophenyl-3-(4-methoxyphenyl)-1-phenyl-2-pyrazoline (38). Prepared as yellow plates in 79\% yield, mp $124-125{ }^{\circ} \mathrm{C} ;{ }^{1} \mathrm{H}-\mathrm{NMR}(\delta): 3.02\left(1 \mathrm{H}, \mathrm{dd}, \mathrm{J}=6.1,17.9 \mathrm{~Hz}, 4-\mathrm{H}_{\text {trans }}\right), 3.85$ (3H, s, MeO), 3.97 (1H, dd, J = 12.8, 17.9 Hz, 4-H cis $\left._{2}\right), 5.59$ (1H, dd, J =6.1, $\left.12.8 \mathrm{~Hz}, 5-\mathrm{H}\right), 6.80$ 7.70 (m, 12x arom. H); ${ }^{13} \mathrm{C}-\mathrm{NMR}(\delta): 40.9,54.3,59.8,111.9,112.9,118.1,124.1,126.2,126.8$, 127.4, 128.0, 128.6, 131.4, 132.7, 137.0, 143.5, 146.1, 159.3; Anal. Calcd. for $\mathrm{C}_{22} \mathrm{H}_{18} \mathrm{Cl}_{2} \mathrm{~N}_{2} \mathrm{O}$ : C, 66.51; H, 4.57; N, 7.05. Found: C, 66.61; H, 4.62; N, 7.14\%.

5-(2,6-Dichlorophenyl)-3-(4-methoxyphenyl)-1-phenyl-2-pyrazoline (39). Prepared as pale yellow needles in $75 \%$ yield, mp $178-179{ }^{\circ} \mathrm{C}$; ${ }^{1} \mathrm{H}-\mathrm{NMR}(\delta): 3.34(1 \mathrm{H}, \mathrm{dd}, \mathrm{J}=9.1,17.6 \mathrm{~Hz}, 4-$ $\left.\mathrm{H}_{\text {trans }}\right), 3.80\left(1 \mathrm{H}, \mathrm{dd}, \mathrm{J}=13.2,17.6 \mathrm{~Hz}, 4-\mathrm{H}_{\text {cis }}\right), 3.88(3 \mathrm{H}, \mathrm{s}, \mathrm{MeO}), 6.04(1 \mathrm{H}, \mathrm{dd}, \mathrm{J}=9.1,13.2 \mathrm{~Hz}$, 5-H), 6.77-7.74 (m, 12x arom. H); ${ }^{13} \mathrm{C}-\mathrm{NMR}(\delta): 40.2,55.4,60.1,113.0,114.1,118.9,125.4$, $127.2,128.5,128.9,129.2,130.8,135.1,136.0,145.0,146.5,160.2$; Anal. Calcd. for $\mathrm{C}_{22} \mathrm{H}_{18} \mathrm{Cl}_{2} \mathrm{~N}_{2} \mathrm{O}: \mathrm{C}, 66.51 ; \mathrm{H}, 4.57 ; \mathrm{N}, 7.05$. Found: $\mathrm{C}, 66.43 ; \mathrm{H}, 4.51 ; \mathrm{N}, 7.14 \%$.

5-(3,4-Dichlorophenyl)-3-(4-fluorophenyl)-1-phenyl-2-pyrazoline (40). Obtained as pale yellow plates in $76 \%$ yield, mp $131-132{ }^{\circ} \mathrm{C}$; ${ }^{1} \mathrm{H}-\mathrm{NMR}(\delta): 3.11(1 \mathrm{H}, \mathrm{dd}, \mathrm{J}=5.3,17.6 \mathrm{~Hz}$, 4$\left.\mathrm{H}_{\text {trans }}\right), 3.88\left(1 \mathrm{H}, \mathrm{dd}, \mathrm{J}=11.4,17.6 \mathrm{~Hz}, 4-\mathrm{H}_{\text {cis }}\right), 5.77(1 \mathrm{H}, \mathrm{dd}, \mathrm{J}=5.3,11.4 \mathrm{~Hz}, 5-\mathrm{H}), 6.82-7.77$ $(\mathrm{m}, 12 \mathrm{x}$ arom. $\mathrm{H}) ;{ }^{13} \mathrm{C}-\mathrm{NMR}(\delta): 43.5,63.6,105.3,113.4,115.5,115.9,119.7,125.2,127.5$, 127.6, 127.9, 128.1, 129.0, 130.3, 131.2, 142.8, 144.5; Anal. Calcd. for $\mathrm{C}_{21} \mathrm{H}_{15} \mathrm{FCl}_{2} \mathrm{~N}_{2}$ : C, 65.47; H, 3.92; N, 7.27. Found: C, 65.58; H, 3.95; N, 7.38\%.

3-(4-Chlorophenyl)-5-(2,4-dichlorophenyl)-1-phenyl-2-pyrazoline (41). Isolated as yellow needles in $81 \%$ yield, mp $156-157{ }^{\circ} \mathrm{C} ;{ }^{1} \mathrm{H}-\mathrm{NMR}(\delta): 3.03\left(1 \mathrm{H}, \mathrm{dd}, \mathrm{J}=5.9,17.4 \mathrm{~Hz}, 4-\mathrm{H}_{\text {trans }}\right)$, $3.98\left(1 \mathrm{H}, \mathrm{dd}, \mathrm{J}=11.9,17.4 \mathrm{~Hz}, 4-\mathrm{H}_{\mathrm{cis}}\right), 5.66(1 \mathrm{H}, \mathrm{dd}, \mathrm{J}=5.9,11.9 \mathrm{~Hz}, 5-\mathrm{H}), 6.82-7.70$ (m, 12x arom. H); ${ }^{13} \mathrm{C}$ - NMR $(\delta): 41.6,61.0,113.2,119.7,124.1,126.9,127.9,128.3,128.8,129.1$, 129.8, 130.9, 132.4, 134.0, 134.6, 137.7, 144.0, 145.9; Anal. Calcd. for $\mathrm{C}_{21} \mathrm{H}_{15} \mathrm{Cl}_{3} \mathrm{~N}_{2}$ : C, 62.78; H, 3.76; N, 6.97. Found: C, 62.88; H, 3.70; N, 6.88\%.

3-(4-Chlorophenyl)-5-(2,6-dichlorophenyl)-1-phenyl-2-pyrazoline (42). Prepared as pale yellow needles in 71\% yield, mp $193-194{ }^{\circ} \mathrm{C} ;{ }^{1} \mathrm{H}-\mathrm{NMR}(\delta): 3.33(1 \mathrm{H}, \mathrm{dd}, \mathrm{J}=9.3,17.8 \mathrm{~Hz}, 4-$ $\left.\mathrm{H}_{\text {trans }}\right), 3.80\left(1 \mathrm{H}, \mathrm{dd}, \mathrm{J}=12.7,17.8 \mathrm{~Hz}, 4-\mathrm{H}_{\text {cis }}\right), 6.12(1 \mathrm{H}, \mathrm{dd}, \mathrm{J}=9.3,12.7 \mathrm{~Hz}, 5-\mathrm{H}), 6.79-7.71$ (m, 12x arom. H); ${ }^{13} \mathrm{C}-\mathrm{NMR}(\delta): 39.7,60.1,113.1,119.4,126.8,128.5,128.7,128.9,129.3$, 
130.8, 131.1, 134.2, 135.0, 135.6, 144.3, 145.2; Anal. Calcd. for $\mathrm{C}_{21} \mathrm{H}_{15} \mathrm{Cl}_{3} \mathrm{~N}_{2}$ : C, 62.78; $\mathrm{H}, 3.76$, N, 6.97. Found: C, 62.64; H, 3.80; N, 7.04\%.

3-(4-Chlorophenyl)-5-(3,4-dichlorophenyl)-1-phenyl-2-pyrazoline (43). Isolated as yellow needles in $77 \%$ yield, mp $136-137{ }^{\circ} \mathrm{C} ;{ }^{1} \mathrm{H}-\mathrm{NMR}(\delta): 3.09\left(1 \mathrm{H}, \mathrm{dd}, \mathrm{J}=5.9,17.7 \mathrm{~Hz}, 4-\mathrm{H}_{\text {trans }}\right)$, $3.86\left(1 \mathrm{H}, \mathrm{dd}, \mathrm{J}=11.9,17.7 \mathrm{~Hz}, 4-\mathrm{H}_{\text {cis }}\right), 5.27(1 \mathrm{H}, \mathrm{dd}, \mathrm{J}=5.9,11.9 \mathrm{~Hz}, 5-\mathrm{H}), 6.82-7.70$ (m, 12x arom. H); ${ }^{13} \mathrm{C}$ - NMR $(\delta): 43.3,63.7,105.4,113.2,125.3,126.9,127.8,128.9,129.8,130.5$, $131.8,132.9$, 133.6, 134.6, 139.5, 142.7, 144.3, 145.6, 151.1; Anal. Calcd. for $\mathrm{C}_{21} \mathrm{H}_{15} \mathrm{Cl}_{3} \mathrm{~N}_{2}$ : C, 62.78; H, 3.76; N, 6.97. Found: C, 62.85; H, 3.82; N, 7.08\%.

\section{Acknowledgments}

The present study was sponsored by the Hungarian National Research Foundation (Grant No. OTKA T049468), to which our gratitude is expressed. The technical assistance of Mrs. M. Nagy is highly appreciated.

\section{References}

1. Wiley, R. H., Ed. Pyrazoles, Pyrazolines, Pyrazolidines, Indazoles and Condensed Rings; In The Chemistry of Heterocyclic Compounds; Weissberger, A.., Ed.; Interscience Publishers: New York, 1967; Vol. 22, p 180.

2. Elguero, J. In Comprehensive Heterocyclic Chemistry II, Katritzky, A. R.; Rees, C. W.; Scriven, E. F., Eds; Pergamon Press: Oxford, 1996; Vol. 3, p1.

3. Lévai, A. Khim. Geterotsikl. Soedin. 1997, 747.

4. Lévai, A. J. Heterocycl. Chem. 2002, 39, 1.

5. Ramalingham, K.; Thyvekikakath, G. X.; Berlin, K. D.; Chesnut, R. W.; Brown, R. A.; Durham, N. N.; Ealick, A. E.; van der Helm, D. J. Med. Chem. 1977, $20,847$.

6. Brown, R. E.; Shavrel Jr., J. US Patent 1972, 3,624,102; Chem. Abstr. 1972, 76, 59618.

7. Lombardino, J. G.; Otterness, I. G. J. Med. Chem. 1981, 24, 830.

8. Fischer, E.; Knövenagel, O. Ann. Chem. 1887, 239, 194.

9. Raiford, L. C.; Peterson, W. J. J. Org. Chem. 1936, 1, 544.

10. Raiford, L. C.; Gundy, G. V. J. Org. Chem. 1938, 3, 265.

11. Raiford, L. C.; Manley, R. H. J. Org. Chem. 1940, 5, 590.

12. Ried, W.; Dankert, G. Chem. Ber. 1957, 90, 2707.

13. Wiley, R. H.; Jarboe, C. H.; Hayes, F. N.; Hansbury, E.; Nielsen, J. T.; Callahan, P. X.; Sellars, M. C. J. Org. Chem. 1958, 23, 732.

14. Sammour, A. E. A. Tetrahedron 1964, 20, 1067.

15. Bhatnagar, I; George, M. V. Tetrahedron 1968, 24, 1293.

16. Aubagnac, J. L; Elguero, J.; Jacquier, R. Bull. Soc. Chim. Fr. 1969, 3292. 
17. Weber, F. G.; Brosche, K.; Seedorf, C.; Rinow, A. Monatsh. Chem. 1969, 100, 1924.

18. Joshi, M. G.; Wadodkar, K. N. Indian J. Chem. 1981, 20B, 1090.

19. Sharma, T. C.; Pawar, S. R.; Reddy, N. J. Acta Chim. Hung. 1983, 112, 159.

20. Dhar, D. N.; Raghunathan, R. Indian J. Chem. 1984, 23B, 1187.

21. Orlov, V. D.; Aziz, M. A.; Mchedlov-Petrosyan, N. O.; Asoka, P. K. D. Khim. Geterotsikl. Soedin. 1985, 1511.

22. Sachchar, S. P.; Singh, A. K. J. Indian Chem. Soc. 1985, 62, 142.

23. Lévai, A.; Szöllősy, Á; Tóth, G. J. Chem. Research (S) 1985, 392.

24. Tóth, G.; Szöllősy, Á.; Lóránd, T.; Kónya, T.; Szabó, D.; Földesi, A.; Lévai, A. J. Chem. Soc., Perkin Trans. 2 1989, 319.

25. Szöllősy, Á.; Tóth, G.; Lóránd, T.; Kónya, T.; Aradi, F.; Lévai, A. J. Chem. Soc., Perkin Trans. 2 1991, 489.

26. Andotra, C. S.; Khajuria, J.; Singh, G. B.; Singh, S. J. Indian Chem. Soc. 1993, 70, 266.

27. Bilgin, A. A.; Palaska, E.; Sunal, R.; Gümüsel, B. Pharmazie 1994, 49, 67.

28. Mishriky, N.; Asaad, F. M.; Ibrahim, Y. A.; Girgis, A. S. Pharmazie 1996, 51, 544.

29. Lévai, A. J. Heterocycl. Chem. 1998, 35, 13.

30. Lévai, A. Heterocycl. Commun. 1999, 5, 151.

31. Dighade, S. R.; Chincholkar, M. M. Asian J. Chem. 2001, 13, 1606.

32. Wang, P.; Onozawa-Komatsuzaki, N.; Himeda, Y.; Sugihara, H.; Arakawa, H., Kasuga K. Tetrahedron Lett. 2001, 42, 9199.

33. Manna, F.; Chimenti, F.; Bolasco, A.; Secci, D.; Bizzarri, B.; Befani, O.; Turini, P.; Mondovi, B.; Alcaro, S.; Tafi, A. Bioorg. Med. Chem. Lett. 2002, 12, 3629.

34. Lévai, A.; Patonay, T.; Silva, A. M. S.; Pinto, D. C. G. A.; Cavaleiro, J. A. S. J. Heterocycl. Chem. 2002, 39, 751.

35. Lévai, A. Heterocycl. Commun. 2003, 9, 287.

36. Lévai, A.; Silva, A. M. S.; Pinto, D. C. G. A.; Cavaleiro, J. A. S.; Alkorta, I.; Elguero, J.; Jekö, J. Eur. J. Org. Chem. 2004, 4672.

37. Lyle, R. E.; Paradis, L. P. J. Am. Chem. Soc. 1955, 77, 667.

38. Dawey, W.; Gwilt, J. R. J. Chem. Soc. 1957, 1008.

39. Jha, B. C.; Amin, G. C. Tetrahedron 1958, 2, 241.

40. Cavaleiro, J. A. S.; Elguero, J.; Jimeno, M. L.; Silva, A. M. S. Chem. Lett. 1991, 445. 